\title{
Notices / Avis
}

\section{Nominations for Officers of the Canadian Political Science Association}

In accordance with the provisions of article 8.2 of the Constitution of The Canadian Political Science Association, the Nominating Committee invites nominations for the office of President-Elect and of five (5) Members-at-Large on the Board of Directors.

You are reminded that each nomination requires the signatures of two proposers, and that it must be accompanied by a statement of acceptance signed by the nominee, as well as by the nominee's 100-word curriculum vitae.

All participants in these procedures must be paid-up members of the Association. You are further reminded that voting is by mail ballot.

Nominations must be received before January 31,1989 , by The Secretary-Treasurer, Canadian Political Science Association, University of Ottawa, Ottawa, Canada K1N 6N5.

\section{Candidatures au Bureau de direction de l'Association canadienne de science politique}

Selon l'article 8.2 de la Constitution, le Comité des candidatures invite les membres de l'Association canadienne de science politique à présenter des candidat(e)s au poste de Président(e)-désigné(e) ainsi qu'à cinq (5) postes au Bureau de direction. (Nous élisons 5 des 10 membres du Bureau de direction par année.)

Il faut que chaque candidature soit accompagnée de l'appui de deux membres, d'un avis d'acceptation signé par la personne choisie, ainsi que d'un curriculum vitae de celle-ci d'environ 100 mots.

Pour être candidat(e) ou pour présenter une candidature, il faut être membre en règle de l'Association. Veuillez prendre note que l'élection se fera par courrier.

Les mises en candidatures doivent parvenir avant le 31 janvier, 1989, au Secrétaire-trésorier, Association canadienne de science politique, Université d'Ottawa, Ottawa, Canada K1N 6N5. 